04

\title{
Прохождение плазменных сгустков через поперечный магнитный барьер
}

\author{
(C) А.М. Бишаев, ${ }^{1}$ М.Б. Гавриков, ${ }^{2}$ М.В. Козинцева, ${ }^{1}$ В.В. Савельев ${ }^{2,3}$ \\ ${ }^{1}$ Московский технологический университет МИРЭА, \\ 119454 Москва, Россия \\ ${ }^{2}$ Институт прикладной математики им. М.В. Келдыша РАН, \\ 125047 Москва, Россия \\ ${ }^{3}$ Национальный исследовательский ядерный университет «МИФИ», \\ 115409 Москва, Россия \\ e-mail: ssvvvv@rambler.ru; bishaev@mirea.ru
}

(Поступило в Редакцию 26 апреля 2017 г.)

\begin{abstract}
Инжекция плазменного сгустка в мультипольную ловушку может применяться для создания плазмы в объеме ловушки. Инжекция сгустка в ловушку типа токамак может рассматриваться как дополнительный инструмент управления процессом нагрева плазмы и для доставки топлива в центральную зону термоядерного реактора. В обоих случаях инжекция производится перпендикулярно магнитному полю ловушки. Теория, эксперимент и математическое моделирование показали, что глубина проникновения плазменного сгустка в поперечное магнитное поле пропорциональна энергии сгустка и обратно пропорциональна магнитному давлению и площади поперечного сечения сгустка. Полученные результаты дают возможность оценить параметры плазменного сгустка, при которых он будет захвачен в ловушку. Это позволило оптимизировать процесс захвата сгустка в ловушку.
\end{abstract}

DOI: 10.21883/JTF.2018.01.45476.2309

\section{Введение}

Создание высокотемпературной плазмы в мультипольной магнитной ловушке [1] является ключевой проблемой при разработке таких ловушек. В МИРЭА этот процесс осуществлялся с помощью инжекции плазменного сгустка в ловушку [2]. Эксперименты [3] показали, что температура ионов плазмы после захвата сгустка в ловушку примерно в 3 раза меньше, чем энергия иона в сгустке. Поэтому температуру ионов в ловушке на уровне $1000 \mathrm{eV}$ можно получить, захватив в ловушку плазменный сгусток с энергией ионов $3000 \mathrm{eV}$. Процесс остановки такого сгустка в магнитном поле ловушки требует специального рассмотрения. Магнитное поле мультипольной магнитной ловушки можно рассматривать как магнитный барьер. Поэтому для осуществления захвата сгустка в ловушку необходимо исследовать процесс прохождения сгустка через поперечный магнитный барьер. Задачей настоящей работы является определение соотношения между параметрами магнитного барьера (величиной поперечного магнитного поля в барьере, протяженностью барьера) и параметрами плазменного сгустка (энергией, размерами), при которых сгусток остановится в барьере.

\section{1. Остановка плазменного сгустка в поперечном магнитном барьере}

При прохождении плазменного сгустка через поперечное магнитное поле считается, что сгусток не должен проходить через поле, если магнитное давление больше, чем скоростной напор плазменного сгустка $[4,5]$ :

$$
B^{2} / 2 \mu_{0} \geq \rho V^{2}
$$

где $B-$ величина магнитной индукции в барьере, $\mu_{0}-$ магнитная постоянная, $\rho-$ плотность плазмы в сгустке, $V$ - скорость плазменного сгустка поперек направления магнитного поля. Однако эксперименты по изучению прохождения плазменных сгустков через магнитный барьер [4,5] показали, что остановка (отсечка) сгустка магнитным полем наступала, если магнитное давление превышало скоростной напор сгустка по крайней мере на порядок. Оценка величины магнитного поля, которое необходимо для остановки сгустка в барьере по соотношению (1), значительно расходится с экспериментальными данными. Это можно объяснить тем, что (1) является соотношением между силами, а именно силы давления магнитного поля, действующей на сгусток, и силы, обусловленной скоростным напором сгустка. Равенство этих сил не означает, что скорость сгустка при прохождении барьера становится равной нулю. В настоящей работе процесс прохождения плазменного сгустка через магнитный барьер рассматривается на основании закона сохранения импульса (количества движения), который справедлив даже и при наличии в системе диссипативных процессов. Если сгусток останавливается в магнитном барьере, то импульс, который получает магнитное поле от сгустка (импульс, который воспринимают магнитные катушки), равен начальному количеству движения сгустка

$$
\left(B^{2} / 2 \mu_{0}\right) S \Delta t=\rho V_{0} S L_{p},
$$


где $S$ - площадь поперечного сечения сгустка, $\Delta t-$ время от начала движения сгустка через магнитное поле до его остановки в барьере, $L_{p}$ - длина плазменного сгустка, $V_{0}-$ скорость сгустка перед барьером. При составлении соотношения (2) предполагалось, что магнитное поле в барьере постоянное. Если сгусток останавливается в барьере, пройдя расстояние $L_{b}$, то $\Delta t$ можно оценить как отношение $L_{b}$ к средней скорости движения сгустка в барьере - $\langle V\rangle$. $L_{b}$ будем называть длиной пробега сгустка в барьере. Максимальное значение $L_{b}$ равно длине магнитного барьера. Если длина $L_{b}$ превышает длину магнитного барьера, то сгусток не останавливается в барьере. Величина средней скорости $\langle V\rangle$ зависит от того, как сила со стороны барьера изменяется по мере движения сгустка в нем. Если сила остается постоянной, то $\langle V\rangle=k V_{0}=0.5 V_{0}$. Если сила линейно растет, то $k=0.64$. В результате получается, что сгусток отсекается магнитным полем, если выполняется критерий: магнитное давление, деленное на начальный скоростной напор сгустка, больше отношения длины сгустка к длине пробега сгустка в магнитном барьере, умноженному на коэффициент $k$ :

$$
\left(\frac{B^{2}}{2 \mu_{0}}\right) /\left(\rho V_{0}^{2}\right) \geq k L_{p} / L_{p b} .
$$

В установках по наполнению плазмой магнитных ловушек $[4,5]$ и исследованию взаимодействия плазменного потока с мишенями [6] плазменные сгустки генерируются плазменной пушкой и проходят по плазмоводу. Скорость плазменных сгустков определяется по времени пролета сгустка между магнитными зондами, установленными в плазмоводе. Длина сгустка может быть определена по длительности сигнала на магнитном зонде, умноженной на величину скорости сгустка. Определенная по данным работ [4,5] длина сгустка составила величину 2-3 m, а в [6] она достигает $16 \mathrm{~m}$. Длина магнитного барьера (максимальная длина пробега сгустка в барьере) в работах $[4,5]$ не превышала $20 \mathrm{~cm}$. В соответствии с критерием (3) остановка сгустка в барьере должна происходить при превышении магнитного давления в барьере над скоростным напором сгустка в 5-10 раз. Этот результат согласуется с данными, полученными в $[4,5]$. Здесь следует заметить, что в экспериментальных установках длина сгустка значительно превышает длину магнитного барьера, а также, что понятие длина сгустка точно не определено, так как сгусток состоит из быстрой головной части и медленного длинного хвоста. Поэтому при оценке длины сгустка всегда присутствует некоторая неопределенность.

\section{2. Описание экспериментов}

На стенде для исследования мультипольных магнитных ловушек в МТУ МИРЭА [2,3] были проведены эксперименты по прохождению плазменных сгустков через поперечное магнитное поле. Схема эксперимента

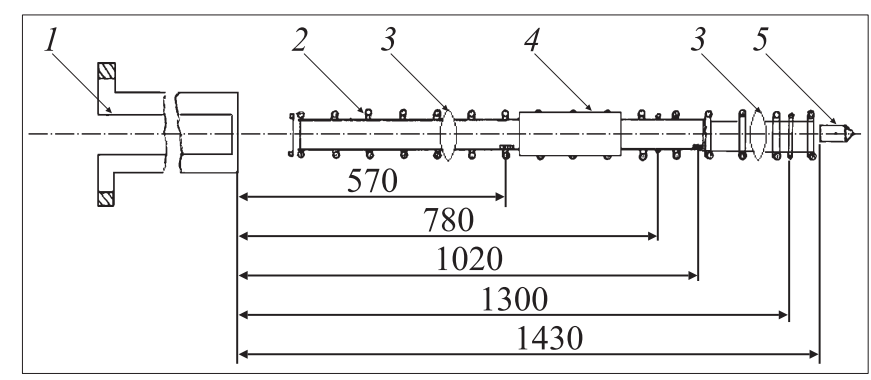

Рис. 1. Схема эксперимента.

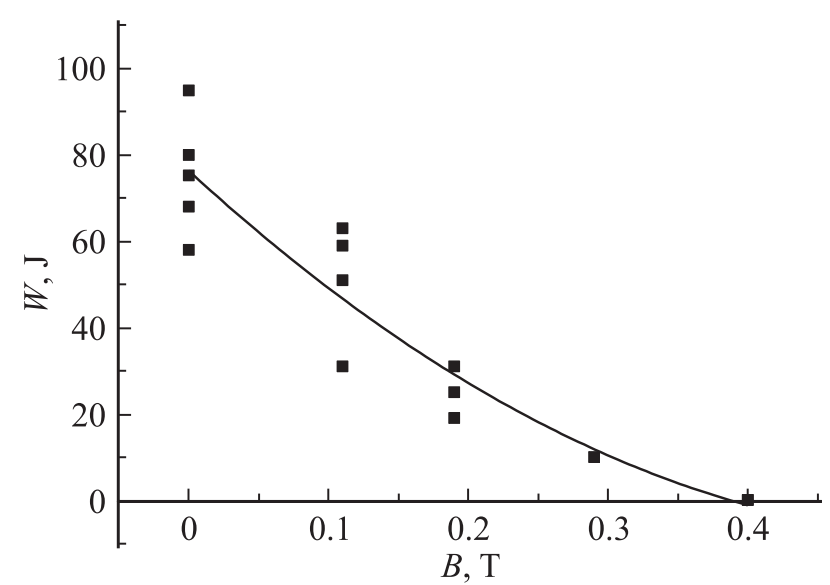

Рис. 2. Зависимость энергии сгустка $W$, прошедшего магнитный барьер, от величины поля в барьере $B$.

приведена на рис. 1. Плазменные сгустки генерировались плазменной пушкой 1 и поступали в плазмовод 2. В средней части плазмовода установлены две прямоугольные катушки 4 системы магнитного барьера (отсечки), которые создавали поперечное к направлению распространения сгустка магнитное поле на длине $0.2 \mathrm{~m}$. Скорость и длина плазменного сгустка определялись по сигналам с двух магнитных зондов 3, установленных в плазмоводе. В конечном сечении плазмовода сгусток попадал в калориметр 5. В эксперименте снималась зависимость величины сигнала с калориметра от величины магнитного поля в магнитном барьере. Из этих экспериментов была определена величина магнитного поля, при которой сгусток полностью отсекался. Результаты приведены на рис. 2. Они показывают, что сигнал с калориметра становится равным нулю при величине магнитного поля в барьере $\sim 0.4 \mathrm{~T}$. Таким образом, сгустки полностью отсекаются этим полем. Определенная по сигналам с магнитных зондов длина сгустков составила величину $\sim 2 \mathrm{~m}$ и отношение $L_{p} / L_{b}=10$. Полученные результаты показывают: для остановки сгустков давление магнитного поля должно превышать динамический напор при $k=0.5-0.64$ в 5-7 раз. Однако определение длины сгустков по длительности сигналов с магнитных зондов весьма неточно. В эксперименте более надежно измеряется величина энергии сгустка калориметром. 
Поэтому выражение (3) было преобразовано с целью исключения из него $L_{p}$. Величина энергии сгустка $W$ равна $W=\frac{m V^{2}}{2}=L_{p} S \rho V^{2} / 2$, или $\rho V^{2}=2 W / L_{p} S$. Подставляя величину динамического напора в (3), получим

$$
L_{b}=(2 k W / S) /\left(B^{2} / 2 \mu_{0}\right) \text {. }
$$

То есть длина пробега сгустка в барьере пропорциональна энергии сгустка и обратно пропорциональна площади поперечного сечения сгустка и квадрату величины магнитного поля в барьере. Для проверки соотношения (4) была определена площадь поперечного сечения сгустка. Для этого в плазмовод помещалась гребенка магнитных зондов. Результаты измерений показали, что сгусток полностью заполнял сечение плазмовода. Из рис. 2 видно, что величина энергии сгустка $W$ лежит в пределах 59-95J. Средняя энергия сгустка составляет величину $72 \mathrm{~J}$. Диаметр сгустка, равный диаметру плазмовода, составляет величину $0.1 \mathrm{~m}$. Если считать, что $k=0.64$, то по (4) $L_{b}=0.19 \mathrm{~m}$. Этот результат удовлетворительно совпадает с длинной магнитного барьера $0.2 \mathrm{~m}$, полученной по расчетам и измерениям конфигурации магнитного поля, которое использовалось в экспериментах. Дополнительные подтверждения правомерности предложенного подхода к определению длины пробега сгустка в барьере были получены при математическом моделировании процесса прохождения сгустка через поперечный магнитный барьер. Результаты такого моделирования приводятся в разд. 3.

\section{3. Численное моделирование прохождения сгустка через магнитный барьер}

Точная постановка задачи о движении плазменного сгустка поперек внешнего магнитного поля очень громоздкая и сложная (например, в рамках уравнений Власова-Максвела). В настоящей работе численное моделирование проводилось в предположении, что сгусток представляет собой недеформируемое тело с бесконечной проводимостью (твердый сверхпроводник). В [6] экспериментально показано, что при движении мощных плазменных сгустков по плазмоводу магнитное поле внутри сгустка равно нулю и деформация сгустка незначительна. Эти наблюдения подтверждают правомерность такой постановки задачи. Поэтому полученные при численном моделировании зависимости длины пробега сгустка в барьере $L_{b}$ от энергии сгустка $W$, величины магнитного поля в барьере $B$, площади поперечного сечения сгустка $S$ могут рассматриваться как подтверждение соотношения (4).

Численное моделирование производилось в двумерном плоском приближении в прямоугольной области $X_{\min }<x<X_{\max }, Y_{\min }<y<Y_{\min }$ (рис. 3). Сгусток представляет собой бесконечное по оси $z$ тело. В этой плоскости сечение сгустка имеет форму прямоугольника

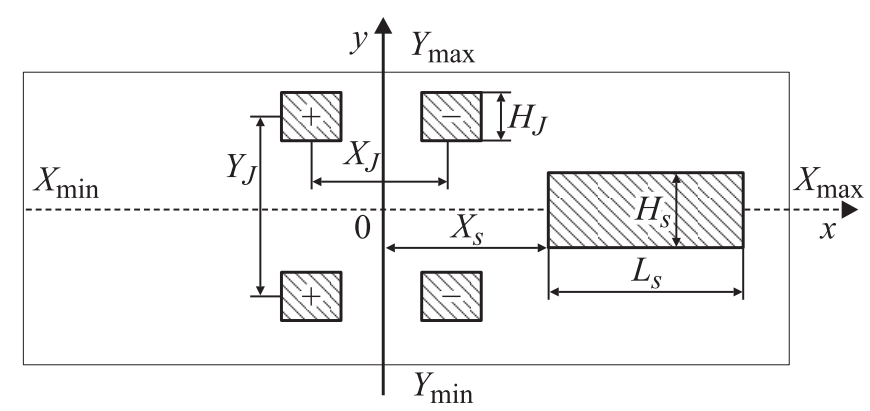

Рис. 3. Геометрия области решения задачи.

с размерами $L_{s} \times H_{s}$. Положение сгустка по оси $X$ определяется расстоянием $X_{s}$ от начала координат. Задача ставится в вакуумной области, т.е. в области внешней к сгустку. Магнитное поле создается токами $J$, текущими вдоль оси $z$ по четырем проводникам квадратного сечения $H_{J} \times H_{J}$. Во всех проводниках токи $J$ по модулю одинаковы, отличаясь знаком в левой и правой парах.

Задача об определении внешнего барьерного магнитного поля в двумерной модели сводится к решению уравнения Пуассона для $z$-компоненты потенциала магнитного поля $A(x, y)$

$$
\frac{\partial^{2} A}{\partial x^{2}}+\frac{\partial^{2} A}{\partial y^{2}}=-\frac{4 \pi}{c} j(x, y), \quad B_{x}=\frac{\partial A}{\partial y}, \quad B_{y}=\frac{\partial A}{\partial x}
$$

с соответствующими граничными условиями на границе области. А именно на внешних границах области задаются значения потенциала или нулевой нормальной производной. Таким образом, верхняя и нижняя границы предполагаются хорошо проводящими, а на боковых границах — „свободные“ условия.

$$
\begin{gathered}
A(x, y)=A_{0}=\mathrm{const}, \quad y=Y_{\min }, Y_{\max }, \\
\frac{\partial A}{\partial x}=0, x=X_{\min }, X_{\max },
\end{gathered}
$$

где $j(x, y)$ - кусочно-постоянная функция отличная от нуля в области сечений четырех проводников, где $j(x, y)= \pm J / H_{J}^{2}$. Чтобы учесть наличие сверхпроводящего тела, нужно еще поставить граничные условия на его поверхности. Сделаем это так, чтобы при большом расстоянии тела от начала координат $\left|X_{s}\right|$ поле было бы малым $(\mathbf{B} \approx 0)$, как и в его отсутствии. Поэтому полагаем на поверхности тела

$$
A(x, y)=A_{0} .
$$

На рис. 4 представлены примеры результатов расчета потенциала магнитного поля при различных расстояниях сгустка от барьера $X_{s}$. Параметры этого расчета

$X_{\min }=-100 \mathrm{~cm}, X_{\max }=110 \mathrm{~cm}, Y_{\min }=-25 \mathrm{~cm}$,

$Y_{\max }=25 \mathrm{~cm}, H_{s}=10 \mathrm{~cm}, L_{s}=40 \mathrm{~cm}$,

$H_{J}=5 \mathrm{~cm}, J=700 \mathrm{kA}$.

Сгусток „перемещается“ вдоль оси $X$ слева направо. Для каждого положения сгустка (например, с шагом по $X_{s}$, 

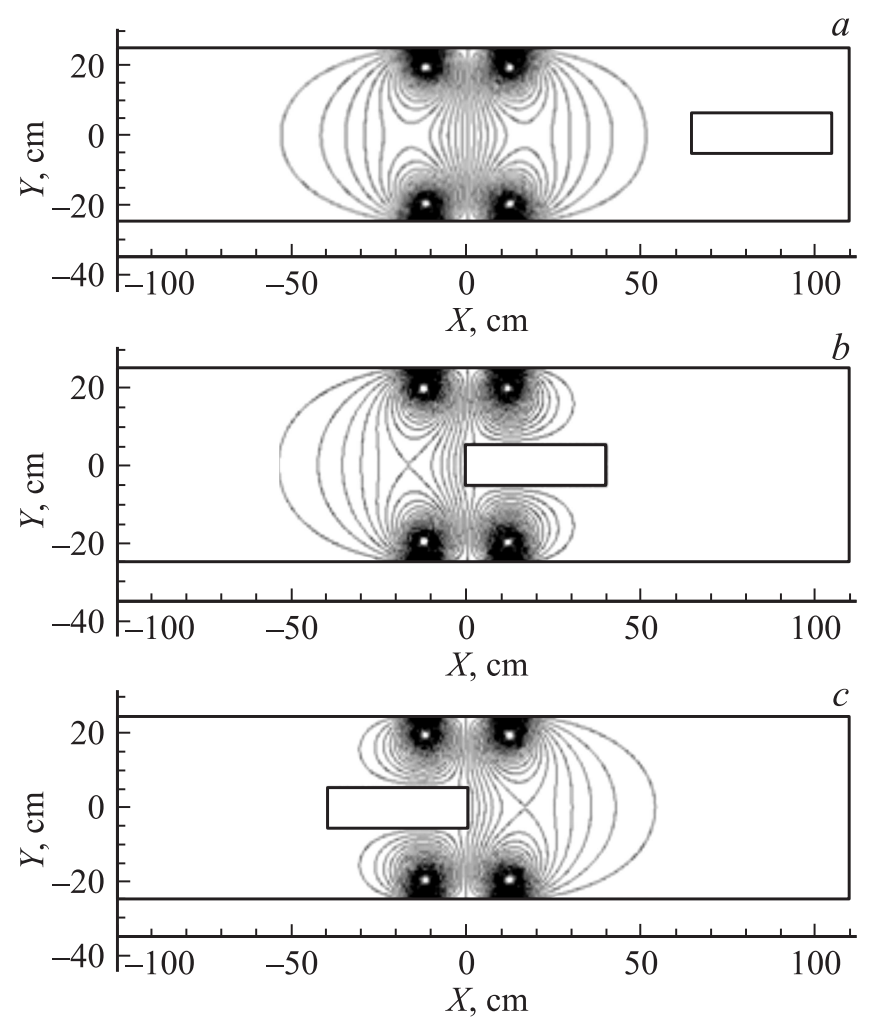

Рис. 4. Распределения магнитного поля (линии $A(x, y)=$ = const) при $X_{s}=65(a), 0(b)-40 \mathrm{~cm}(c)$.

равным $5 \mathrm{~cm}$ ) рассчитывается конфигурация магнитного поля $A(x, y)$. А затем вычисляются величины $X-$ компоненты силы $F\left(X_{s}\right)$, действующей на единицу длины сгустка в направлении оси $Z$, полный ток $J_{p l}\left(X_{s}\right)$ и потенциальная энергия единицы длины сгустка $U(x)$

$$
\begin{gathered}
F_{x}=-\oint \frac{\mathbf{B}^{2}}{8 \pi} n_{x} d l, \quad J_{p}=\frac{c}{4 \pi} \oint B_{t} d l, \\
U(x)=-\int^{x} F\left(X_{s}\right) d X_{s} .
\end{gathered}
$$

Здесь контурные интегралы берутся по „поверхности“ сгустка. В положении, когда середина сгустка проходит через точку $x=0$, конфигурация магнитного поле становится опять симметричной относительно этой точки. Поэтому при вычислении интегралов достаточно ограничиться „Половиной“ задачи. Расчеты велись по двум методикам: разностной (со стандартной аппроксимацией двумерного оператора Лапласа) и методом конечных элементов на треугольной сетке. В дальнейших расчетах будут меняться только следующие параметры: $J$ ток в проводниках и $H_{s}$ - ширина сгустка (размер сгустка по оси $y$ ). Расчеты (параметры расчета такие же, как и на рис. 4) показывают (рис. 5), что при прохождении барьера в сгустке возбуждается электрический ток $J_{l}$, который исчезает после прохождения сгустком магнитного барьера. На рис. 5 приведены также результаты расчета силы $F$, действующей на сгусток и потенциальная энергия сгустка $U$. Эти расчеты показали, что сила, действующая на сгусток, возрастает до тех пор, пока задняя часть сгустка не окажется в барьере. Потенциальная энергия сгустка $U$ в барьере возрастает, достигает максимума, а затем убывает. Если энергия сгустка $W$ меньше максимального значения потенциальной энергии, то сгусток останавливается в барьере. Зависимость потенциальной энергии сгустка от $X$ (профиль $U(x)$ ) позволяет определить координату $x=X_{b}$, при которой сгусток останавливается. Координата $X_{b}$ соответствует значению потенциальной энергии, равной величине энергии сгустка: $U(x)=W$. Поэтому предложенная математическая модель позволяет определить зависимости $X_{b}$ от величины $W, H_{s}$ и $J$. С этой целью были рассчитаны (рис. 6,a) профили потенциальной энергии $U(x)$ в зависимости от размера сгустка по оси $y-H_{s}$ при фиксированном токе $J$ в проводниках и профили потенциальной энергии $U(x)$ в зависимости от величины тока $J$ при фиксированной ширине сгустка $H_{s}$ (рис. $\left.6, b\right)$. Используя результаты расчетов на рис. $6, a$, получены значения $X_{b}$ по координатам точек пересечения линии $U=\mathrm{const}=70 \mathrm{~J}$ с профилями потенциальной энергии для трех значений $H_{s}$. По профилям, приведенным на рис. $6, b$, были определены значения $X_{b}$ точек линии пересечения $U=$ const $=70 \mathrm{~J}$ с профилями потенциальной энергии для трех значений $J$. По профилю $U$ для $J=800 \mathrm{kA}$ были определены значения $X_{b}$ для четырех значений $U=70,80,90,100 \mathrm{~J}$. Полученные результаты представлены на рис. 7. На этом рисунке для линий, соединяющих полученные из расчетов точки, был выбран линейный закон аппроксимации. Отклонение $X_{b}$ от их значений, аппроксимированных по линейному закону, не превышает $10 \%$. Из рис. 7 следует, что $X_{b}$ пропорциональна величине энергии сгустка $W$ (рис. 7,a), обратно пропорциональна ширине сгустка $H_{s}$ (рис. $7, b)$ и квадрату тока в проводниках $J^{2}$ (рис. $\left.7, c\right)$. Координата остановки сгустка в барьере $X_{b}$ (по определению) должна быть пропорциональна длине пробега сгустка в барьере $L_{b}$. Следовательно, для подтверждения

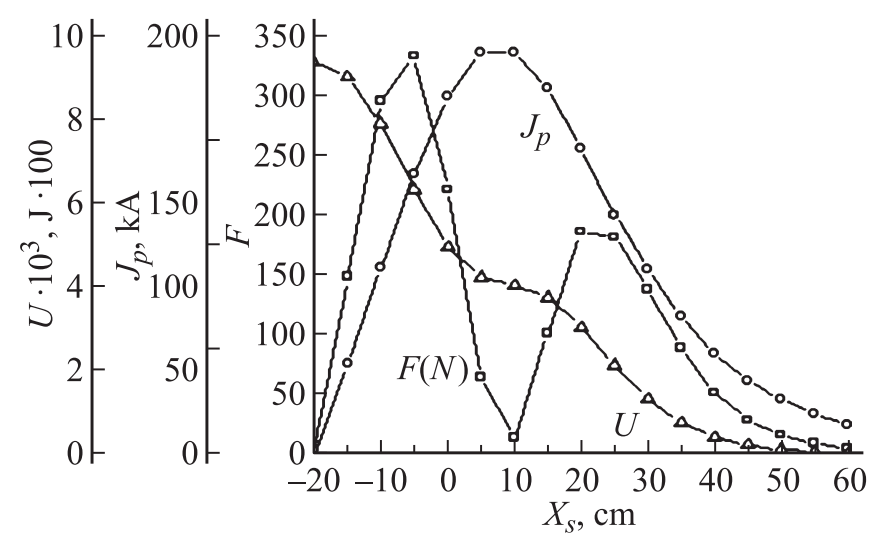

Рис. 5. Зависимость величины потенциальной энергии сгустка $U$, тока по поверхности сгустка $J_{p}$ и силы, действующей на сгусток $F$, от положения сгустка в барьере $X_{s}$. 

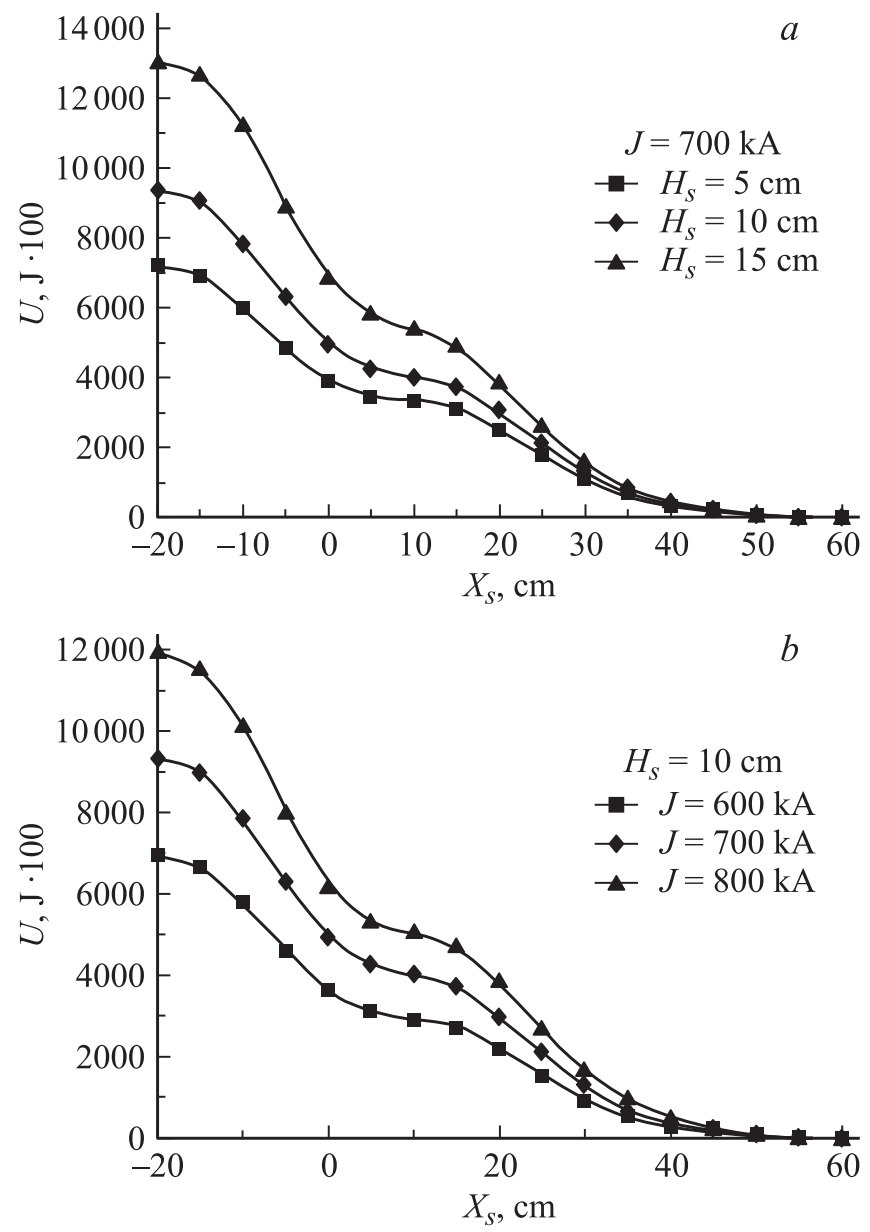

Рис. 6. Профили потенциальной энергии: $a-$ в зависимости от размера сгустка по оси $y H_{s}$ при фиксированной величине тока в проводниках $J=700 \mathrm{kA} ; b-$ в зависимости от величины тока в проводниках при фиксированной ширине сгустка $H_{s}=10 \mathrm{~cm}$.

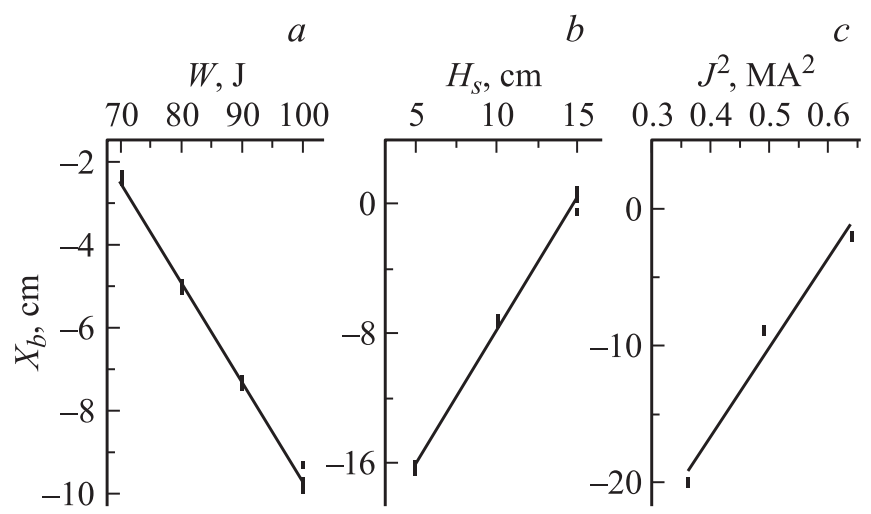

Рис. 7. Зависимость координаты остановки сгустка в барьеpe $X_{b}$ от: $a$-энергии сгустка $W, b$ - ширины сгустка $H_{s}, c-$ квадрата тока в проводниках $J^{2}$.

соотношения (4) нужно найти зависимость $X_{b}$ от величины $W, S$ и $B(4)$. Двумерная постановка задачи математического моделирования прохождения сгустка через барьер рассматривает часть сгустка, имеющего единицу длины в направлении оси $z$. При такой постановке площадь сгустка $S$ пропорциональна его ширине $H_{s}$. Величина тока в проводниках $J$ пропорциональна величине магнитного поля в барьере $B$. Поэтому, учитывая, что $X_{b}$ пропорциональна $L_{b}$, полученные результаты подтверждают то, что $L_{b}$ пропорциональна величине энергии сгустка $W$, обратно пропорциональна площади поперечного сечения сгустка $S$ и квадрату величины магнитного поля в барьере $B^{2}$. Таким образом, результаты математического моделирования не противоречат соотношению (4).

\section{Заключение}

Полученное на основании закона сохранения импульса соотношение для оценки длины проникновения плазменного сгустка в магнитный барьер хорошо согласуется с результатами работ $[3,4]$, а также с экспериментальными и теоретическими результатами, представленными в разд. 3 и 4 настоящей работы. Полученное выражение (4) позволяет оценить параметры сгустка для прохождения магнитного барьера. Эта зависимость свидетельствует о возможности применять инжекцию плазменных сгустков не только в мультипольные ловушки, но и в ловушки типа токамак, что экспериментально было подтверждено в [7]. В настоящее время плазменные сгустки, получаемые с помощью плазменных пушек, имеют весьма высокие параметры. Так, плазменный инжектор, описанный в работе [5], генерирует плазменные сгустки с энергией $W=42 \mathrm{~kJ}$ и площадью поперечного сечения $3 \cdot 10^{-2} \mathrm{~m}^{2}$. Оценка длины проникновения сгустка с такими параметрами в магнитное поле термоядерного реактора ИТЭР [8], равное $5.3 \mathrm{~T}$, составляет величину $22 \mathrm{~m}$, что более чем в 3 раза превосходит большой радиус реактора.

Исследование выполнено при поддержке Российского научного фонда (грант № 16-11-10278).

\section{Список литературы}

[1] Морозов А.И., Савельев В.В. // УФН. 1998. Т. 168. № 11. C. $1153-1194$.

[2] Бишаев А.М., Бугрова А.И., Гавриков М.Б., Козинщева М.В., Липатов А.С., Савельев В.В., Сигов А.С., Смирнов П.Г., Тарелкин И.А., Храмцов П.П. // ЖТФ. 2013. Т. 83. Вып. 4. С. 34-40.

[3] Бишаев А.М., Бугров Г.Э., Десятсков А.В., Козинцева М.В., Огарков П.В., Сазонов П.Г., Гавриков М.Б., Савельев В.В. // Российский технологический журнал. 2015. № 2 (7). С. 101-112. Электронное сетевое издание ISSN 2500-316X. Электронный ресурс. Режим доступа: https://rtj.mirea.ru/upload/medialibrary/7ff/09-bishaev.pdf

[4] Андрюхина Э.Д., Шпигель И.С. // ЖТФ. 1965. Т. 35. № 7. C. $1242-1251$. 
[5] Васильев В.И., Житлухин А.М., Струнников В.М., Хамидуллин Ф.Р. Энциклопедия низкотемпературной плазмы. T. 1Х-3. Радиационная плазмодинамика. Гл. 3. Физика, техника и применение мощных импульсных плазменных ускорителей. М.: Янус-К, 2008. С. 244-278.

[6] Позняк И.М., Архипов Н.И., Карелов С.В., Сабронов В.М., Топорков Д.А. // ВАНТ. Сер. Термоядерный синтез. 2014. Т. 37. Вып. 1. С. 70-79.

[7] Абрамова К.Б., Воронин А.В., Гусев В.К. и др. // Физика плазмы. 2005. Т. 31. № 9. С. 1-9.

[8] Миямото K. Основы физики плазмы и управляемого синтеза / Под общ. ред. В.Д. Шафранова. М.: Физматгиз, 2007. C. $333-335$. 